\title{
Complex Interactions of Lovastatin with 10 Chemotherapeutic Drugs: A Rigorous Evaluation of Synergism and Antagonism
}

\section{Kaitlyn K. Gilman}

University of Arizona

Seungmin Han

University of Arizona

\section{Young-Wook Won}

University of Arizona

Charles W. Putnam ( $\nabla$ cwp@u.arizona.edu )

University of Arizona Arizona Health Sciences Center https://orcid.org/0000-0002-4487-9358

\section{Research article}

Keywords: Statins, chemotherapy, drug interaction, synergism, antagonism, Loewe model, Combenefit, dual drug assay, tamoxifen

Posted Date: June 2nd, 2020

DOl: https://doi.org/10.21203/rs.3.rs-30659/v1

License: (a) (i) This work is licensed under a Creative Commons Attribution 4.0 International License. Read Full License

Version of Record: A version of this preprint was published at BMC Cancer on April 6th, 2021. See the published version at https://doi.org/10.1186/s12885-021-07963-w. 


\section{Abstract \\ Background}

Evidence bearing on the role of statins in the prevention and treatment of cancer is confounded by the diversity of statins, chemotherapeutic agents and cancer types included in the numerous published studies; consequently, the adjunctive value of statins with chemotherapy remains uncertain.

\section{Methods}

We assayed lovastatin in combination with each of ten commonly prescribed chemotherapy drugs in highly reproducible in vitro assays, using an indifferent cellular substrate, Saccharomyces cerevisiae. Cell density $\left(\mathrm{OD}_{600}\right)$ data were analyzed for synergism and antagonism using the Loewe additivity model implemented with the Combenefit software.

\section{Results}

Four of the ten chemotherapy drugs - tamoxifen, doxorubicin, methotrexate and rapamycin - exhibited net synergism with lovastatin. The remaining six agents (5-fluorouracil, gemcitabine, epothilone, cisplatin, cyclophosphamide and etoposide) compiled neutral or antagonistic scores. Distinctive patterns of synergism and antagonism, often coexisting within the same concentration space, were documented with the various combinations, including those with net synergism scores.

\section{Conclusions}

The interactions of tamoxifen, doxorubicin, methotrexate and rapamycin with lovastatin suggest clinical utility and merit further exploration in cell lines and animal models. No less importantly, strong antagonistic interactions between certain agents and lovastatin argue for a cautious, data-driven approach before adding a statin to chemotherapeutic regimens. We also urge awareness of adventitious statin usage by patients entering cancer treatment protocols.

\section{Background}

From antiquity [1] to the present day cancer has plagued humanity; in 2018 cancer claimed an estimated 9.6 million lives, one in six deaths worldwide [2]. Despite the many consequential improvements in cancer treatment, there remains a clinical imperative to identify novel therapies with improved efficacy and diminished toxicity. In this context, statins [3-hydroxy-3-methylglutaryl-CoA (HMG-CoA) reductase inhibitors], the most commonly prescribed class of pharmaceuticals worldwide, have engendered promise and drawn scrutiny. 
In 1971 Akira Endo isolated the first statin progenitor, citrinin [3]. Soon it was shown to be an inhibitor of HMG-CoA reductase [4], the rate limiting step in the mevalonate pathway. Although citrinin proved to be nephrotoxic, in 1976 the Endo laboratory [5, 6] and a British group [7] independently isolated ML-236B, also called compactin. Eleven years later, the FDA approved the first commercial statin: naturally derived lovastatin. Subsequently six statins, including two semi-synthetic and four synthetic formulations, have entered the marketplace and represent primary therapy for the prevention of cardiovascular disease. Today, an estimated 30 million people worldwide take statins.

Within a few years of the introduction of statins, however, concerns regarding their safety emerged, notably an associated increase in non-cardiac mortality [8]. Animal studies suggested that they might be carcinogenic: when given statins at doses equivalent to those commonly prescribed in humans, rats developed lymphomas and carcinomas of the liver, stomach, lung, and thyroid [9]. Consequently, large randomized clinical trials were undertaken to evaluate not only the efficacy of statins but also any associated risk of cancer. Ironically, by demonstrating reduced incidences of colorectal carcinoma, prostate cancer and melanoma [10], these studies were the first to indicate that statins might prevent cancer. To date, preclinical and clinical data suggest chemopreventive effects of statins against a variety of cancers including those of the breast [11], colon [12], lung [13], liver [14], pancreas [15], and prostate [16].

Several lines of evidence had also suggested that statins might have value in the treatment of cancer: statins modulate the mevalonate pathway [17], which ultimately modifies the posttranslational processing of proteins involved in cell cycle control; cancer cells exhibit increased synthesis, receptor mediated uptake, and degradation of cholesterol (reviewed in [18]); and, disrupted cholesterol homeostasis has been demonstrated in various tumor models (see, for example, [19]). In 1998, Matar, et al. [20], published a landmark study: a short course of lovastatin in rats inhibited primary fibrosarcoma growth and diminished the size and number of experimentally induced lung metastases. Subsequently, a number of publications have supported the notion that statins exert anticancer activity through mevalonate-dependent and independent mechanisms, as recently reviewed [17, 21].

Disappointingly, statins alone have not proven effective as anticancer therapy; however, there is evidence that statins might potentiate the effects of anti-cancer drugs [22]. A recent systematic review and metaanalysis by Mei et al. [23] (which included 95 studies, 1,111,407 patients and over 18 cancer types) compared statin users to individuals not taking statins. Patients also receiving statins experienced a $30 \%$ reduction in all-cause mortality, a 40\% reduction in cancer-specific mortality, and prolonged progressionfree, recurrence-free, and disease-free survivals. Yet soon after the publication of Mei's analysis, Farooqui et al. [24] published a systematic review and meta-analysis of 10 randomized controlled studies involving 1,881 individuals with stage 3 or 4 cancers, in which statin use did not improve progression-free or overall survival.

Reaching a sound assessment of the value of statins as adjuncts to conventional chemotherapy from clinical trials is confounded by the numbers of different drugs - both statins and chemotherapeutic agents - featured in the various investigations, and the multiplicity of cancer types treated. Consequently, we Loading [MathJax]/jax/output/CommonHTML/jax.js ind chemotherapeutic drug interactions by rigorously assaying 
a single statin (lovastatin) in combination with each of ten FDA-approved chemotherapeutic agents with a variety of mechanisms of action. Because our objective was to compare the ten drug pairings with each other, it was essential to use an indifferent cellular substrate, rather than a human cancer cell line which because of its inherent cellular and genetic biases might favor or disadvantage a particular drug pair. Instead, we chose as the cellular substrate the exceptionally well-studied model organism, Saccharomyces cerevisiae [25]. There is ample precedent for utilizing budding yeast in research related to cancer and its therapies [25-27]. Many of its genes have human orthologs [28] and a number of cell signaling pathways now recognized as critical to oncogenesis were first identified and/or extensively studied in yeast. Importantly, S. cerevisiae has been utilized in a number of pharmacologic studies and high throughput screens [29-31], including ones specifically focused on anticancer drug research (reviewed in [32]).

In order to better represent the genetic diversity emblematic of malignancies, we created a balanced pool of heterozygous deletion strains of essential genes. (The barcoded pool also provides a resource for investigating genetically-driven resistance to drug treatments [33].) We configured a 96-well microplate assay compatible with the Combenefit dual-drug interaction software [34]; cell concentration data, read spectrophotometrically, were submitted to rigorous statistical analysis for synergistic or antagonistic interactions calculated according to the Loewe additivity model [35], included in the Combenefit package.

Our data demonstrate that combining lovastatin with conventional chemotherapeutic agents results in drastically different interactions, ranging from strong synergism to profound antagonism, often within the same concentration space. Of the ten chemotherapeutic drugs, only four (tamoxifen, doxorubicin, methotrexate, and rapamycin) exhibited net synergism with lovastatin; two drugs (gemcitabine and 5fluorouracil) had neutral scores; and four (epothilone, cisplatin, cyclophosphamide, and etoposide) displayed net antagonism.. As proof of principle, two of the drug combinations, tamoxifen/lovastatin and cisplatin/lovastatin, were further evaluated in human cancer cell lines. The results in cell lines were generally accordant with the data obtained with $S$. cerevisiae but with variations in the patterns of synergism and antagonism between individual cell lines, even of the same cancer type.

\section{Methods}

\section{Saccharomyces cerevisiae heterozygous deletion pool}

The complete collection of $S$. cerevisiae heterozygous diploid essential gene deletion strains was obtained from ThermoScientific. A balanced pool was created, aliquoted and frozen (see Additional file 1: Methods). For each set of assays, $10 \mathrm{ml}$ of synthetic complete media with $2 \%$ dextrose (SCD) was inoculated with 10 $\mu \mathrm{l}$ of pooled cells and incubated overnight in a shaking water bath at $29^{\circ} \mathrm{C}$. Before using in assays, the cells were diluted with $S C D$ to an $\mathrm{OD}_{600}$ reading of $0.7-0.85$.

\section{Chemotherapeutic And Statin Drugs}


The following drugs were obtained from Cayman Chemical (Ann Arbor, MI, USA): cisplatin (cis Diammineplatinum hydrochloride), cyclophosphamide (hydrate), epothilone B, etoposide, 5-fluorouracil, lovastatin (lovastatin hydroxy acid, sodium salt), rapamycin, and tamoxifen. Compounds obtained from Millipore Sigma included: doxorubicin hydrochloride, gemcitabine hydrochloride, and methotrexate.

\section{Saccharomyces cerevisiae dose response curves}

In order to determine suitable concentration ranges for the dual drug dilution assays, dose response curves were compiled for each of the 11 drugs, using a range of concentrations based upon published experimental data in S. cerevisiae, where available. The methodology is described in detail in Additional file 1: Methods.

\section{Saccharomyces cerevisiae dual drug dilution assay}

Dual drug assays were performed in 96-well tissue culture plates using a uniform format compatible with the input requirements of the Combenefit software package (see later). In each assay, lovastatin was paired with a single chemotherapeutic drug. A schematic diagram of the plate configuration is shown in Additional file 2: Figure S1, and the methodology for the assay is described in detail in Additional file 1: Methods. The concentration ranges for each drug tested are given in Table 1. Crosswise, serial $70 \%$ dilutions create a matrix of 49 unique dual-drug combinations; the plates also include individual drug dose responses along with positive (cells only) and negative (SCD only) control wells. Plates are sealed and incubated with shaking at $29^{\circ} \mathrm{C}$. Cell densities are read using a plate reader $\left(\mathrm{OD}_{600}\right)$, after two and three days of incubation. Each experiment was conducted a minimum of 3 times; the numbers of biologic replicates are given in the figure legends. 
Table 1

Concentration ranges of the ten chemotherapeutic drugs and lovastatin in the S. cerevisiae assays. The chemotherapy drugs were chosen in part based upon their diverse mechanisms of action

\begin{tabular}{|lll|}
\hline Drug & Principal mechanism of action & $\begin{array}{c}\text { Concentration Range } \\
(\mu \mathrm{M})\end{array}$ \\
\hline Cisplatin & DNA crosslinking & $12.4-105$ \\
\hline Cyclophosphamide & Alkylating agent which binds to DNA & $49.4-420$ \\
\hline Doxorubicin & Intercalates DNA, blocking replication & $24.7-210$ \\
\hline Epothilone & Binds to microtubules, blocking cell division & $1.1-9.3$ \\
\hline Etoposide & Topoisomerase II inhibitor & $0.165-1.4$ \\
\hline 5-Fluorouracil & Antimetabolite: blocks thymidylate synthetase & $0.12-1.05$ \\
\hline Gemcitabine & $\begin{array}{l}\text { Antimetabolite: incorporated into DNA in place of } \\
\text { dCTP }\end{array}$ & $14.4-123$ \\
\hline Methotrexate & Antimetabolite: binds to dihydrofolate reductase & $8.24-70.0$ \\
\hline Rapamycin & mTOR inhibitor & $0.33-2.80$ \\
\hline Tamoxifen & Selective estrogen receptor modulator (SERM) & $24.7-210$ \\
\hline Lovastatin & Competitive inhibitor of HMG-CoA reductase & $12.7-108$ \\
\hline
\end{tabular}

\section{Assays In Human Cell Lines}

Crosswise, dual-dilution format assays were also performed using human cancer cell lines as substrate. The cell lines, obtained from ATCC, included: A549 (adenocarcinoma, lung), HCC827 (adenocarcinoma, lung), HT-29 (colorectal adenocarcinoma), MCF7 (metastatic adenocarcinoma, breast, estrogen, progesterone and growth factor receptor expressing), MDA-MB-231 (metastatic adenocarcinoma, breast, triple negative for receptor expression), and SK-BR-3 (metastatic adenocarcinoma, breast, HER2 overexpressing). The cells were cultured according to the corresponding ATCC protocols.

The dual drug crosswise dilution matrix assays were performed in a similar format as the S. cerevisiae experiments and likewise included a plate blank, individual drug dose responses, and cells-only positive controls (see Additional file 1: Methods, for a detailed description of the assay procedure). The plates were incubated for two days at $37^{\circ} \mathrm{C}$; cell viability was assessed with the MTT assay (Sigma Aldrich), according to the manufacturer's protocol. 
In the S. cerevisiae assays, each plate was scrutinized for outlier values by the Grubb's test, implemented with the XLSTAT software package. When an outlier was identified, it was removed and replaced as described in Additional file 1: Methods. Of note, no more than one outlier per column or row was removed. After reconciling outliers, the mean of the positive (cells-only) control wells was calculated and set to a value of 1 ; the plate data were normalized to this value.

The Combenefit software package (http://sourceforge.net/projects/combenefit/) calculates and displays the synergism-antagonism distributions and computes a variety of metrics from the distributions. Although the software renders three models (Bliss, HAS, and Loewe), we chose the Loewe additivity model as the most suitable (see Discussion) because it allows for the possibility that the two drugs have interacting modes of action [36]. The Loewe model determines the degree of interaction by comparing the experimental concentration space to a reference concentration space calculated from the individual doseresponse curves for the two drugs; thus, these must be included in each assay (Additional file 2: Figure S1). The dose response curve for each of the chemotherapeutic drugs is computed by the software from all biologic replicates of that drub combination (see Figs. 2a,b; 3a,b; 4a,b; 5a,d; 7a,b,d,e,g,h,j,k). Dose response curves for lovastatin were also included in every assay; to maximize the reliability of the lovastatin doseresponse curves, we pooled the lovastatin dose-response data from all ten dual drug sets (separately for days two and three). The mean dose response data so obtained for lovastatin were utilized in all ten experimental analyses.

After the Combenefit software compares the experimental surface to the reference surface, it assigns a percentage score to each cell in the matrix; statistical significance is then ascertained using the onesample t test [34]. If the test ascribes significance to a given cell, the cell is colored appropriately to highlight its synergy or antagonism (see, for example, Fig. 2d).

\section{Results}

\section{Evaluation of statin plus chemotherapeutic drug pairs in S. cerevisiae}

Sorting of lovastatin/chemotherapeutic drug pairs using the global metric, "SUM_SYN_ANT". Most drug pairs demonstrated significant synergism and/or antagonism, depending upon the relative concentrations of the two drugs. Therefore, to broadly categorize the ten drug pairs, we exploited the global metrics generated by the Combenfit software. For this purpose, "SUM_SYN_ANT", defined as the "sum of synergy and antagonism observed in concentration logarithmic space" ([34], Supplementary material), was the most useful. Applying this metric, drug pairs with a net score of $>2.0$ were considered "synergistic" (four drug pairs), those with scores in the range of +2.0 to -2.0 , as "neutral" (two drug pairs), and pairs with scores <-2.0, as "antagonistic" (four drug pairs), Fig. 1a. With most drug combinations, the results obtained after two and three days of incubation were similar although the analyses on day three trended toward less synergism and/or greater antagonism, as illustrated by a comparison of the "SUM_SYN_ANT" scores calnulatad fartho twin dave (Eir $1 \mathrm{~h}$ ) Thatimg noteworthy exceptions, methotrexate and rapamycin, are Loading [MathJax]/jax/output/CommonHTML/jax.js 
discussed below. When the data obtained on day three were congruent with the results on day two, only the latter are presented in this section; the day three data can otherwise be found in Additional file 2.

Chemotherapeutic drugs exhibiting net synergism with lovastatin. Based on the metric SUM_SYN_ANT on day two and the criteria just described, tamoxifen (score, +9.36) doxorubicin $(+5.43)$, methotrexate $(+2.93)$ and rapamycin $(+2.16)$ each achieved net synergism when paired with lovastatin (Fig. 1a; Additional file 2, Table S1).

Tamoxifen, which earned the highest composite score (+9.36) on day two (Fig. 1a), also exhibited the greatest maximum synergy, +53.6 , (Fig. $1 \mathrm{c}$ and Fig. 2 d). A score of +53.6 represents about a $54 \%$ increase in effectiveness over that predicted if the two drugs were simply additive. The synergism between lovastatin and tamoxifen was evident over a broad range of concentrations of lovastatin but was narrowly confined to two concentrations of tamoxifen, 50.4 and $72 \mu \mathrm{M}$ (Fig. 2d). Remarkably, a concentration of 72 uM of tamoxifen paired with 75.5 uM lovastatin resulted in a score of $+26(p<.0001)$, yet a further 0.70 dilution of lovastatin (108 uM) at the same concentration of tamoxifen resulted in significant antagonism (score - 26, p < 0.05), Fig. 1d and Fig. 2d. The experimental combination dose response surface, with an overlay of synergy levels, is shown in Fig. 2c; this plot - a depiction of efficacy - illustrates the substantial inhibition of growth achieved within the synergistic space. Finally, we note that the synergy and antagonism matrix for tamoxifen plus lovastatin on day three (Additional file 2: Figure S2a-d) shares a similar pattern with the day two results.

The interactions of doxorubicin with lovastatin were statistically neutral over more than 80 percent of the concentration space (Fig. 3d); however, this combination earned the second highest composite score, + 5.43 (Fig. 1a; Additional file 2: Table S1) by virtue of the statistically significant synergism at the two highest concentrations of doxorubicin (147 and $210 \mathrm{uM}$ ) and the complete absence of significant antagonistic interactions (Fig. 1d and 3d). As was the case with tamoxifen, the significant synergistic concentrations of lovastatin embraced a wide range (18.1-75.5 uM) when paired with doxorubicin. Synergism was greatest on day 2 with a maximum score of 23.3 (Fig. 1c; Additional file 2: Table S1), trending downward on day 3 (Fig. 1b) but retaining a similar pattern (Additional file 2: Figure S3a-d). Importantly, the efficacy (see Fig. 3c and Additional file 2: Figure S3c) of the synergistic interactions was quite modest.

The SUM_SYN_ANT score for the methotrexate-lovastatin pairing was a modest, but perhaps misleading, + 2.93 (Fig. 1b): statistically significant synergism and antagonism coexisting in the concentration space (Fig. 1c,d and Fig. 4c,d) largely offset each other in the calculation of the global score. Importantly, the lower concentrations of methotrexate interacted synergistically with lovastatin, while the higher methotrexate concentrations interacted antagonistically (Fig. 4d). The two lowest concentrations of methotrexate ( 8.2 and $11.8 \mu \mathrm{M})$ demonstrated statistically significant synergism over the entire concentration range of lovastatin, with maximum synergism (+17.9) evident at the lowest concentration of methotrexate (Fig. 4d). The antipodal concentrations of methotrexate ( 49 and $70 \mu \mathrm{M})$ demonstrated the greatest antagonism (-11), Fig. 1d and Fig. 4d. This pattern persisted on day three but with fading 
statistical significance (Fig. 4e; Additional file 2: Figure S4), causing the global score for methotrexate to flip from net synergism (+2.93) on day two to net antagonism (-3.68) on day three (Fig. 1b).

Rapamycin, in combination with lovastatin, had a modest net score on day two (Fig. 1a) yet it achieved the highest synergism of all drug pairs on day three. In contrast to the trend of increasing antagonism from days 2 to 3 with other pairings, the SUM_SYN_ANT score for rapamycin on day three was nearly eight-fold that on day two $(+16.6$ versus +2.16$)$, Fig. $1 \mathrm{~b}$. As was the case with methotrexate, rapamycin paired with lovastatin exhibited both synergism and antagonism of statistical significance within its concentration spaces (Fig. 5c,f). On day 2, the synergistic and antagonistic interactions clustered toward opposing quadrants in the concentration space: lower concentrations of rapamycin paired with higher concentrations of lovastatin achieved significant synergism while higher concentrations of rapamycin and the two lowest concentrations of lovastatin were antagonistic (Fig. 5c). The maximum scores for synergism and antagonism were +31.7 and -15.6 , respectively (Fig. 1c, d and Additional file 2: Table S1). Reflecting the coexistence of strong synergy and antagonism within the same concentration space, the SUM_SYN_ANT metric was a modest +2.28 . The overall pattern of rapamycin-lovastatin interactions persisted on day three, although synergism greatly increased, spreading further across the concentration space as antagonism receded (Fig. 5f); two-thirds of the cells recorded statistically significant synergism while only one cell retained significant antagonism. A comparison of efficacy afforded by the experimental dose-response space plots (Fig. 5b, e) suggests that the profound growth inhibition conferred by the synergistic interactions was similar on both days, an observation reinforced by comparing the contour maps of the dose response data (Additional file 2: Figure S5). That the effects on cell growth were similar on days two and three, even though the absolute $\mathrm{EC}_{50}$ for rapamycin was increased by about 3.5-fold on day three (Fig. 5 d versus Fig. 5a), suggests that lovastatin somehow prolongs the efficacy of rapamycin.

Chemotherapeutic drug/lovastatin combinations registering neutral or antagonistic SUM_SYN_ANT SCores. None of the six combinations with scores $<+2$ (Fig. 1a) exhibited strong synergism within the interaction matrices (Fig. 6a-f). Three patterns of synergy/antagonism were evident: 5-FU and epothilone had matrices totally devoid of significant interactions (Fig, 6a, c); gemcitabine, cisplatin and cyclophosphamide (Fig. 6b, d, e) registered a mixture of scattered, stronger antagonism interspersed with weaker synergism; and etoposide paired with lovastatin evoked only antagonism (Fig. 6f). The patterns were similar on day three (Additional file 2: Figure S6a-f).

In sum, the interactions of lovastatin with the ten chemotherapeutic drugs were selective: four drug pairs displayed net synergism; however, for six pairs the interactions were either neutral or antagonistic.

\section{Evaluation Of Lovastatin Plus Tamoxifen In Human Cell Lines}

In order to validate the results generated by the yeast model, we submitted two of the drug combinations to further testing in human cell lines. Tamoxifen plus lovastatin was selected because this pairing produced 
principal target of tamoxifen in mammalian cells. Thus, the substantial effects of tamoxifen alone on growth (Fig. 2b), and its strong synergism with lovastatin (Fig. 2d), were quite unexpected. The second combination, lovastatin plus cisplatin, which serves as a surrogate for the antagonistic drug pairings (Fig. 1d), was selected because cisplatin is so widely prescribed for the treatment of a variety of human cancers, including lung, breast, liver, colon, and ovarian carcinomas [37].

Dual drug assays of tamoxifen and lovastatin in human breast and lung cancer cell lines. The human breast cancer cell lines tested included one estrogen receptor (ER) positive, MCF7, and two ER negative, MDA-MB-231 and SK-BR-3 cell lines (see Methods). The results obtained with the ER positive MCF7 cell line are shown in Fig. 7a-c. The global metric SUM_SYN_ANT was +94.4 , the highest obtained in the cell line assays (note that the units and scale of dilutions differ from the assays in yeast). About 30 percent of the concentration space displayed synergism (Fig. 7c) with only one cell indicating statistically significant antagonism. Overall, the pattern is reminiscent of the assays of tamoxifen with lovastatin in S. cerevisiae, see Fig. 2d. The cell line MDA-MB-231 (Fig. 7d-f), from a triple negative breast carcinoma, exhibited both synergism and antagonism, resulting in a global metric of -43.14 . Although about twice as many cells displayed synergism as antagonism, strong antagonism (-50.5) in one cell contributed substantially to the net score. The third breast cancer cell line, ER negative SK-BR-3 (Fig. 7g-i) also displayed a similar pattern of scattered synergism and antagonism, generating a SUM_SYN_ANT score of + 23.7.

When tamoxifen and lovastatin were assayed in the lung adenocarcinoma cell line, HCC827 (Fig. 7j-I), the concentration landscape was weighted toward antagonism, as reflected in the SUM_SYN_ANT score of -130.3. Scattered weak synergy was noted in about 10 per cent of the concentration space.

Dual drug assays of cisplatin and lovastatin in human cancer cell lines. All three cell lines tested - A549, lung adenocarcinoma; HT-29, colon adenocarcinoma; and MCF7, breast carcinoma - exhibited negative (antagonistic) SUM_SYN_ANT scores, with weak maximum synergy and stronger maximum antagonism (Table 2; Additional file 2: Figure S7a-f). The data from the three human cancer cell lines are entirely congruent with the comparable analysis in S. cerevisiae (Table 2).

Table 2

Global metrics of the drug combination lovastatin plus cisplatin in three cell lines: A549 (lung adenocarcinoma), HT29 (colon adenocarcinoma) and MCF7 (breast carcinoma), in comparison to the metrics obtained in similar Saccharomyces cerevisiae analyses.

\begin{tabular}{|lllllllll|}
\hline \multicolumn{3}{|c}{ S. cerevisiae } & A549 & & HT29 & \multicolumn{3}{c|}{ MCF7 } \\
\hline Metrics & Day 2 & Day 3 & Day 2 & Day 3 & Day 2 & Day 3 & Day 2 & Day 3 \\
\hline SUM_SYN_ANT & -2.335 & -3.553 & -7.642 & -9.875 & -5.887 & -3.117 & -33.789 & -13.498 \\
\hline SYN_MAX & 13.895 & 15.698 & 6.014 & 4.812 & 15.346 & 11.252 & 12.558 & 4.922 \\
\hline ANT_MAX & -45.256 & -35.016 & -35.744 & -43.200 & -51.294 & -55.074 & -73.647 & -35.777 \\
\hline
\end{tabular}


Together, the results with lovastatin in combination with cisplatin in three human cell lines and tamoxifen with MCF7 breast cancer cells are concordant with the findings in the S. cerevisiae assays. However, we note marked differences in the interactions of tamoxifen with lovastatin in ER negative breast cancer and lung cancer cell lines (see Discussion).

\section{Discussion}

The data presented in this report should not be taken as - nor was our experimental approach intended to generate - prescriptive guidance for the clinical application of statins as adjuvants to conventional chemotherapeutic agents. Rather, our objective was to illuminate the confounding literature bearing on the adjunctive role of statins in chemotherapy. To that end, we assayed a single statin, lovastatin, paired with each of ten commonly prescribed chemotherapeutic agents, in highly reproducible dilution assays; the data from the assays were submitted to rigorous statistical analysis to identify synergistic and antagonistic interactions, according to the Loewe additivity model. Because the ten chemotherapeutic agents have different mechanisms of action (Table 1), it was not surprising that in combination with lovastatin, they exhibited a spectrum of global interactions, ranging from synergism to neutrality to antagonism (Fig. 1a). In considering the Loewe synergy scores for the various drug interactions, we concur with the view "that any synergy model should be treated as an exploratory ranking statistic for prioritization of the most potent combinations for further evaluation..." [36]. In this context, we identified four chemotherapeutic drugs - tamoxifen, doxorubicin, methotrexate and rapamycin - which from our dual drug studies merit further investigation.

The global metric SUM_SYN_ANT proved useful in identifying the four dual-drug combinations which exhibited strongly synergistic interactions within their concentration spaces. However, undue reliance upon global metrics risks overlooking potentially useful synergistic interactions which are confined to portions of the concentration space, sometimes with coexisting antagonism (see Fig. 4d,e and Fig. 5c,f). Lovastatin in combination with rapamycin proffers a case in point: lower concentrations of rapamycin interact synergistically with higher concentrations of lovastatin yet the converse yields significant antagonism (Fig. 5c,f). A comparison of the interactions of lovastatin with doxorubicin versus methotrexate further illustrate pitfalls of global metrics. The SUM_SYN_ANT score for doxorubicin is higher $(+5.43)$ than for methotrexate (+2.93). However, the synergistic interactions for doxorubicin occur only with the highest two concentrations tested (Fig. 3d), a clinically problematic pattern. By contrast, the synergistic interactions of methotrexate are found with lower concentrations of the chemotherapeutic drug (Fig. 4d,e), an observation suggesting the possibility that adding a statin might not only improve the efficacy of methotrexate but also allow a reduction in its dosage, with attendant mitigation of methotrexate's substantial toxicity.

Together these observations lead to a sobering conclusion: the therapeutic consequences - be they advantageous or detrimental - of a statin/chemotherapeutic drug combination may hinge upon the concentrations of each achieved at the tumor site. Consequently, targeted delivery strategies [38] with precise control of concentration ratios of the two drugs may merit consideration. 
Cognizant of the limitations of global metrics, we gave greater weight in our assessments to the synergy distribution matrices (see, for example, Fig. 2d), in which the statistically significant values are colored for clarity. It should be emphasized that the displayed values - the synergy scores - represent mathematical calculations derived from a comparison of the experimental dose-response space to the reference doseresponse space, in accordance with the Loewe model. On the other hand, the experimental dose-response surface (see, for example, Fig. 2c) portrays the efficacy of the various concentration pairs normalized to the drug-free controls. Both are informative.

Two features of the experimental design merit further discussion. Earlier we set forth our rationale for utilizing the model organism Saccharomyces cerevisiae. Our primary consideration was that the cellular target be an indifferent one, free of the biologic and genetic biases inherent in every cancer cell line; choosing any one human cancer cell line for the assays risked biasing the assays for or against a particular drug. The balanced pool of heterozygously-deleted essential gene strains, which we created in order to have a cellular substrate with consistent genetic diversity, proved to be only modestly more sensitive to the various drugs than the cognate wildtype strain. However, because each deletion is bar coded, the pool provides a useful resource for the analysis of genetic resistance to drug treatments, a study beyond the scope of this report.

A second important consideration in experimental design was the choice of synergy model. The Combenefit software package renders three models (Bliss, HSA and Loewe). The Bliss and Loewe model are arguably the most popular synergy models, but all synergy models have inherent flaws [36, 39]. The probabilistic Bliss model assumes independent but competing drug actions whereas the Loewe additivity model assumes nonindependence; that is, the two drugs may interact with the same targets or pathways [36]. Because of the remarkable pleiotropy of statins (reviewed in detail in [40]), including a number of interactions with a variety of signaling pathways, we posited nonindependence of lovastatin and the individual chemotherapeutic drugs and therefore chose the Loewe model as more appropriate. .

Having demonstrated net synergism of four drug combinations in the yeast model, we selected one tamoxifen and lovastatin - for additional study in human cancer cell lines. This combination exhibited the strongest synergy on day two but also was intriguing because of its unanticipated substantial efficacy in $S$. cerevisiae, which is devoid of estrogen receptors. (Although an estrogen binding protein has been identified in budding yeast [41, 42], the protein demonstrated negligible binding of tamoxifen [41].) The efficacy of tamoxifen alone (Fig. 2b) or paired with lovastatin (Fig. 2c,d) therefore likely results from activation of one or more of tamoxifen's known off-target pathways (reviewed in [43]).

Of the three human breast cancer cell lines assayed, MCF-7 - which possesses estrogen receptors demonstrated strong synergy with lovastatin (Fig. 7c), in a pattern resembling that seen with yeast (Fig. 2d). The other two breast cancer cell lines tested (see Fig. 7f,i), consistently achieved synergy with only the highest concentrations of lovastatin. Together, these observations frame a paradox: the combination of tamoxifen plus lovastatin was strongly synergistic in an organism, S. cerevisiae, devoid of estrogen receptors, yet displayed a similar pattern of strong synergism only in a breast cancer cell line,

Loading [MathJax]/jax/output/CommonHTML/jax.js Perhaps the simplest resolution of this seeming contradiction

Page $12 / 24$ 
is that lovastatin interacts synergistically with tamoxifen only when tamoxifen alone effectively inhibits cell growth, regardless of the cellular mechanism by which that occurs. It therefore seems unlikely - to our disappointment - that a statin will lend efficacy to tamoxifen in triple-negative breast cancer, for example, in which tamoxifen is impotent.

We sought further proof of principle in support of our S. cerevisiae-based experimental design by testing the combination of cisplatin and lovastatin in three human cell lines: A549 (lung adenocarcinoma); HT29 (colon adenocarcinoma) and MCF7 (breast carcinoma). This combination was chosen as a surrogate for the six neutral or antagonistic pairings because it so commonly prescribed for a variety of malignancies. In the yeast model, the combination exhibited strong antagonism (Table 2, Fig. 1a,d; Additional file 2: Fig. S6d). Congruent with our S. cerevisiae data, the metrics for the three cell lines were antagonistic, even more so than those calculated from the yeast assays (Table 2), lending further support for the validity of our model.

Does our study illuminate the contradictory landscape of chemotherapeutic regimens incorporating statins, our purpose as set forth earlier? Perhaps so. In the Background we cited the systemic review and metaanalysis by Farooqui et al., demonstrating that the addition of a statin to conventional therapy failed to improve progression-free or overall survival. Of the ten studies included in their analysis, six incorporated either as the sole chemotherapeutic drug or as a component of a multi-drug regimen - agents which we found to be either neutral or antagonistic: etoposide, cisplatin, gemcitabine, and 5-FU (one protocol included both cisplatin and epirubicin, which is related to doxorubicin). Of the remaining four studies, one specified whole brain irradiation, and three incorporated drugs (afatinib, thalidomide and gefitinib) which we have not assayed. Thus, our data and the Farooqi meta-analysis provide mutually supportive, albeit circumstantial, evidence affirming the lack of efficacy of at least four statin plus chemotherapeutic drug combinations. Unfortunately, the larger systemic review by Mei et al. [23], which demonstrated beneficial effects of statins upon survival in cancer patients, did not specify the individual chemotherapeutic drugs used in the 95 studies included in the analysis.

\section{Conclusions}

The data presented herein are relevant to two clinical scenarios when considering chemotherapeutic protocols. The first scenario is the purposeful inclusion of a statin in a chemotherapeutic drug regimen. If combinations of a statin with any of the four agents achieving synergy in our study - tamoxifen, doxorubicin, methotrexate and rapamycin - weather further scrutiny in in vitro cell line assays and in animal models, clinical trials should be considered.

The second clinical scenario is less obvious but no less important. Patients undergoing chemotherapy may also be receiving a statin, adventitiously co-prescribed for the treatment of elevated lipids or the prevention of cardiovascular disease. In this context, the agents displaying antagonistic interactions with lovastatin gemcitabine, epothilone, cisplatin, cyclophosphamide, and etoposide - generate especial concern. Absent further clinical studies, it would seem prudent for health care providers to weigh the possible

Loading [MathJax]/jax/output/CommonHTML/jax.js e potential risks of decreased chemotherapeutic efficacy.

Page $13 / 24$ 


\section{Abbreviations}

SCD: synthetic complete medium with dextrose

SUM_SYN_ANT - sum of synergy and antagonism observed in concentration logarithmic space

\section{Declarations}

\section{Ethics approval and consent to participate}

Not applicable

\section{Consent for publication}

Not applicable

\section{Availability of data and materials}

The datasets generated and analysed during the current study are available from the corresponding author on reasonable request. The relevant analyses are included in the published article [and its supplementary information files].

\section{Competing interests}

The authors declare that they have no competing interests.

\section{Funding}

National Cancer Institute Award P30CA023074 to the University of Arizona Cancer Center and grant HL138242 (Y-W W) from the National Heart, Lung and Blood Institute.

\section{Authors' contributions}

KG, YWW, and CWP designed the research protocols; KG and SH conducted the experiments; $K G, S H$ and CWP analyzed data; KG and CWP drafted the manuscript; KG, SH, YWW and CWP edited the manuscript. All authors read and approved the final manuscript.

\section{Acknowledgements}


The authors acknowledge David W. Mount, Ph.D., who offered many helpful comments and criticisms throughout the study and critiqued drafts of the manuscript.

\section{References}

1. Binder $M$, Roberts $C$, Spencer N, Antoine D, Cartwright C. On the antiquity of cancer: evidence for metastatic carcinoma in a young man from ancient Nubia (c. 1200 BC). PloS one. 2014;9(3):e90924.

2. https://www.cancerhealth.com/article/world-health-organization-releases-latest-global-cancer-data.

3. Endo A. A historical perspective on the discovery of statins. Proc Jpn Acad Ser B Phys Biol Sci. 2010;86(5):484-93.

4. Endo A, Kuroda M. Citrinin, an inhibitor of cholesterol synthesis. J Antibiot (Tokyo). 1976;29(8):841-3.

5. Endo A, Kuroda M, Tanzawa K. Competitive inhibition of 3-hydroxy-3-methylglutaryl coenzyme A reductase by ML-236A and ML-236B fungal metabolites, having hypocholesterolemic activity. FEBS Lett. 1976;72(2):323-6.

6. Endo A, Kuroda M, Tsujita Y. ML-236A, ML-236B, and ML-236C, new inhibitors of cholesterogenesis produced by Penicillium citrinium. J Antibiot (Tokyo). 1976;29(12):1346-8.

7. Brown AG, Smale TC, King TJ, Hasenkamp R, Thompson RH. Crystal and molecular structure of compactin, a new antifungal metabolite from Penicillium brevicompactum. Journal of the Chemical Society Perkin transactions 1 1976(11):1165-1170.

8. Oliver MF. Might treatment of hypercholesterolaemia increase non-cardiac mortality? Lancet. 1991;337(8756):1529-31.

9. Newman TB, Hulley SB. Carcinogenicity of lipid-lowering drugs. Jama. 1996;275(1):55-60.

10. Demierre MF, Higgins PD, Gruber SB, Hawk E, Lippman SM. Statins and cancer prevention. Nature reviews Cancer. 2005;5(12):930-42.

11. Beckwitt CH, Brufsky A, Oltvai ZN, Wells A. Statin drugs to reduce breast cancer recurrence and mortality. Breast cancer research: BCR. 2018;20(1):144.

12. Hachem C, Morgan R, Johnson M, Kuebeler M, El-Serag H. Statins and the risk of colorectal carcinoma: a nested case-control study in veterans with diabetes. Am J Gastroenterol. 2009;104(5):1241-8.

13. Huang WY, Li CH, Lin CL, Liang JA. Long-term statin use in patients with lung cancer and dyslipidemia reduces the risk of death. Oncotarget. 2016;7(27):42208-15.

14. Singh S, Singh PP, Singh AG, Murad MH, Sanchez W. Statins are associated with a reduced risk of hepatocellular cancer: a systematic review and meta-analysis. Gastroenterology. 2013;144(2):323-32.

15. Zhang Y, Liang M, Sun C, Qu G, Shi T, Min M, Wu Y, Sun Y. Statin Use and Risk of Pancreatic Cancer: An Updated Meta-analysis of 26 Studies. Pancreas. 2019;48(2):142-50.

16. Raval AD, Thakker D, Negi H, Vyas A, Kaur H, Salkini MW. Association between statins and clinical outcomes among men with prostate cancer: a systematic review and meta-analysis. Prostate Cancer 
17. lannelli F, Lombardi R, Milone MR, Pucci B, De Rienzo S, Budillon A, Bruzzese F. Targeting Mevalonate Pathway in Cancer Treatment: Repurposing of Statins. Recent Pat Anti-cancer Drug Discov. 2018;13(2):184-200.

18. Ding X, Zhang W, Li S, Yang H. The role of cholesterol metabolism in cancer. American journal of cancer research. 2019;9(2):219-27.

19. Berger NA. Obesity and cancer pathogenesis. Ann N Y Acad Sci. 2014;1311:57-76.

20. Matar P, Rozados VR, Roggero EA, Scharovsky OG. Lovastatin inhibits tumor growth and metastasis development of a rat fibrosarcoma. Cancer Biother Radiopharm. 1998;13(5):387-93.

21. Vallianou NG, Kostantinou A, Kougias M, Kazazis C. Statins and cancer. Anti-cancer Agents Med Chem. 2014;14(5):706-12.

22. Chae YK, Yousaf M, Malecek MK, Carneiro B, Chandra S, Kaplan J, Kalyan A, Sassano A, Platanias LC, Giles F. Statins as anti-cancer therapy; Can we translate preclinical and epidemiologic data into clinical benefit? Discov Med. 2015;20(112):413-27.

23. Mei Z, Liang M, Li L, Zhang Y, Wang Q, Yang W. Effects of statins on cancer mortality and progression: A systematic review and meta-analysis of 95 cohorts including 1,111,407 individuals. Int J Cancer. 2017;140(5):1068-81.

24. Farooqi MAM, Malhotra N, Mukherjee SD, Sanger S, Dhesy-Thind SK, Ellis P, Leong DP. Statin therapy in the treatment of active cancer: A systematic review and meta-analysis of randomized controlled trials. PloS one. 2018;13(12):e0209486.

25. Botstein D, Fink GR. Yeast: an experimental organism for 21 st Century biology. Genetics. 2011;189(3):695-704.

26. Guaragnella N, Palermo V, Galli A, Moro L, Mazzoni C, Giannattasio S. The expanding role of yeast in cancer research and diagnosis: insights into the function of the oncosuppressors p53 and BRCA1/2. FEMS yeast research. 2014;14(1):2-16.

27. Botstein D, Chervitz SA, Cherry M. Yeast as a Model Organism. 1997, 277(5330):1259-1260.

28. Skrzypek MS, Nash RS, Wong ED, MacPherson KA, Hellerstedt ST, Engel SR, Karra K, Weng S, Sheppard TK, Binkley G, et al. Saccharomyces genome database informs human biology. Nucleic acids research. 2018;46(D1):D736-d742.

29. Smith AM, Ammar R, Nislow C, Giaever G. A survey of yeast genomic assays for drug and target discovery. Pharmacol Ther. 2010;127(2):156-64.

30. Reid RJ, Benedetti P, Bjornsti MA. Yeast as a model organism for studying the actions of DNA topoisomerase-targeted drugs. Biochim Biophys Acta. 1998;1400(1-3):289-300.

31. Sturgeon CM, Kemmer D, Anderson HJ, Roberge M. Yeast as a tool to uncover the cellular targets of drugs. Biotechnology journal. 2006;1(3):289-98.

32. Menacho-Marquez M, Murguia JR. Yeast on drugs: Saccharomyces cerevisiae as a tool for anticancer drug research. Clinical translational oncology: official publication of the Federation of Spanish Oncology Societies of the National Cancer Institute of Mexico. 2007;9(4):221-8. 
33. Giaever G, Nislow C. The Yeast Deletion Collection: A Decade of Functional Genomics. 2014, 197(2):451-465.

34. Di Veroli GY, Fornari C, Wang D, Mollard S, Bramhall JL, Richards FM, Jodrell DI. Combenefit: an interactive platform for the analysis and visualization of drug combinations. Bioinformatics. 2016;32(18):2866-8.

35. S L: The problem of synergy and antagonism of combined drugs. Arzneimittelforshung 1953, 3:285290.

36. Tang J, Wennerberg K, Aittokallio T. What is synergy? The Saariselka agreement revisited. Front Pharmacol. 2015;6:181.

37. Dasari S, Bernard Tchounwou P. Cisplatin in cancer therapy: Molecular mechanisms of action. Eur J Pharmacol. 2014;740:364-78.

38. Piccolo MT, Menale C, Crispi S. Combined anticancer therapies: an overview of the latest applications. Anti-cancer Agents Med Chem. 2015;15(4):408-22.

39. Vlot AHC, Aniceto N, Menden MP, Ulrich-Merzenich G, Bender A. Applying synergy metrics to combination screening data: agreements, disagreements and pitfalls. Drug discovery today. 2019;24(12):2286-98.

40. Gazzerro P, Proto MC, Gangemi G, Malfitano AM, Ciaglia E, Pisanti S, Santoro A, Laezza C, Bifulco M. Pharmacological actions of statins: a critical appraisal in the management of cancer. Pharmacological reviews. 2012;64(1):102-46.

41. Burshell A, Stathis PA, Do Y, Miller SC, Feldman D. Characterization of an estrogen-binding protein in the yeast Saccharomyces cerevisiae. J Biol Chem. 1984;259(6):3450-6.

42. Feldman D, Do Y, Burshell A, Stathis P, Loose DS. An estrogen-binding protein and endogenous ligand in Saccharomyces cerevisiae: possible hormone receptor system. Science. 1982;218(4569):297-8.

43. Radin DP, Patel P. Delineating the molecular mechanisms of tamoxifen's oncolytic actions in estrogen receptor-negative cancers. Eur J Pharmacol. 2016;781:173-80.

\section{Figures}




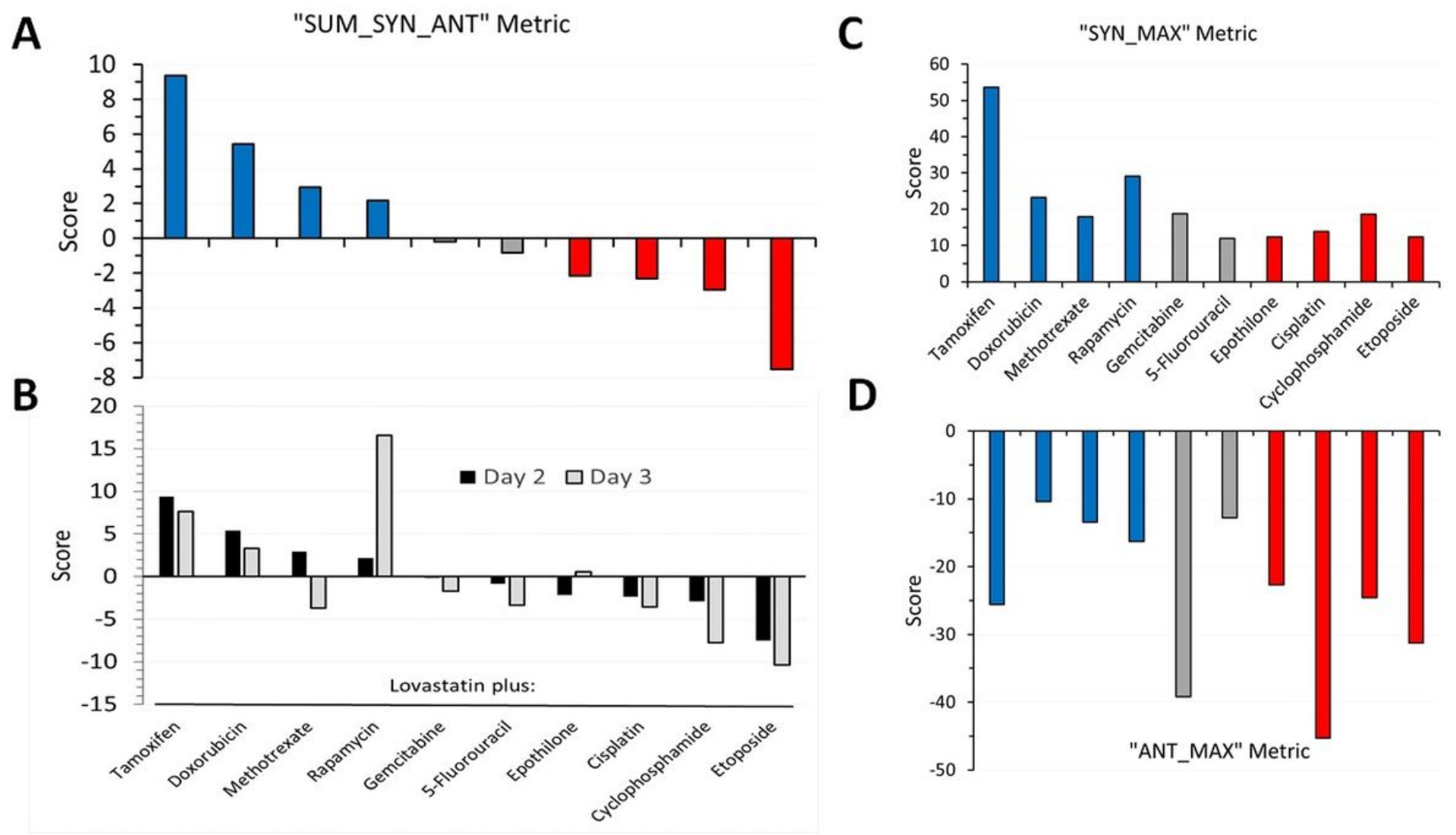

\section{Figure 1}

Graphical representation of three global metrics calculated by the Combenefit software for each of the ten dual-drug assays conducted with the S. cerevisiae heterozygous-deletion pool. A. The SUM_SYN_ANT metric, defined as the "sum of synergy and antagonism observed in concentration logarithmic space" [34], is depicted for the ten drug combinations on day two. The blue bars denote drug combinations with net synergism and the red bars, net antagonism. The grey bars indicate neutral scores. B. A comparison of SUM_SYN_ANT scores on Day 3 versus Day 2. In most, but not all instances (see text), the scores tended toward less synergism or greater antagonism on Day 3. C. The SYN_MAX metric (defined as the "maximum synergy observed") on Day 2; the drug combinations are listed in the same order as in (A). D. The ANT_MAX metric ("maximum antagonism observed") on Day 2. The values in (C) and (D) represent single maximal values, whereas the SUM_SYN_ANT metric encompasses all values within the concentration space. 

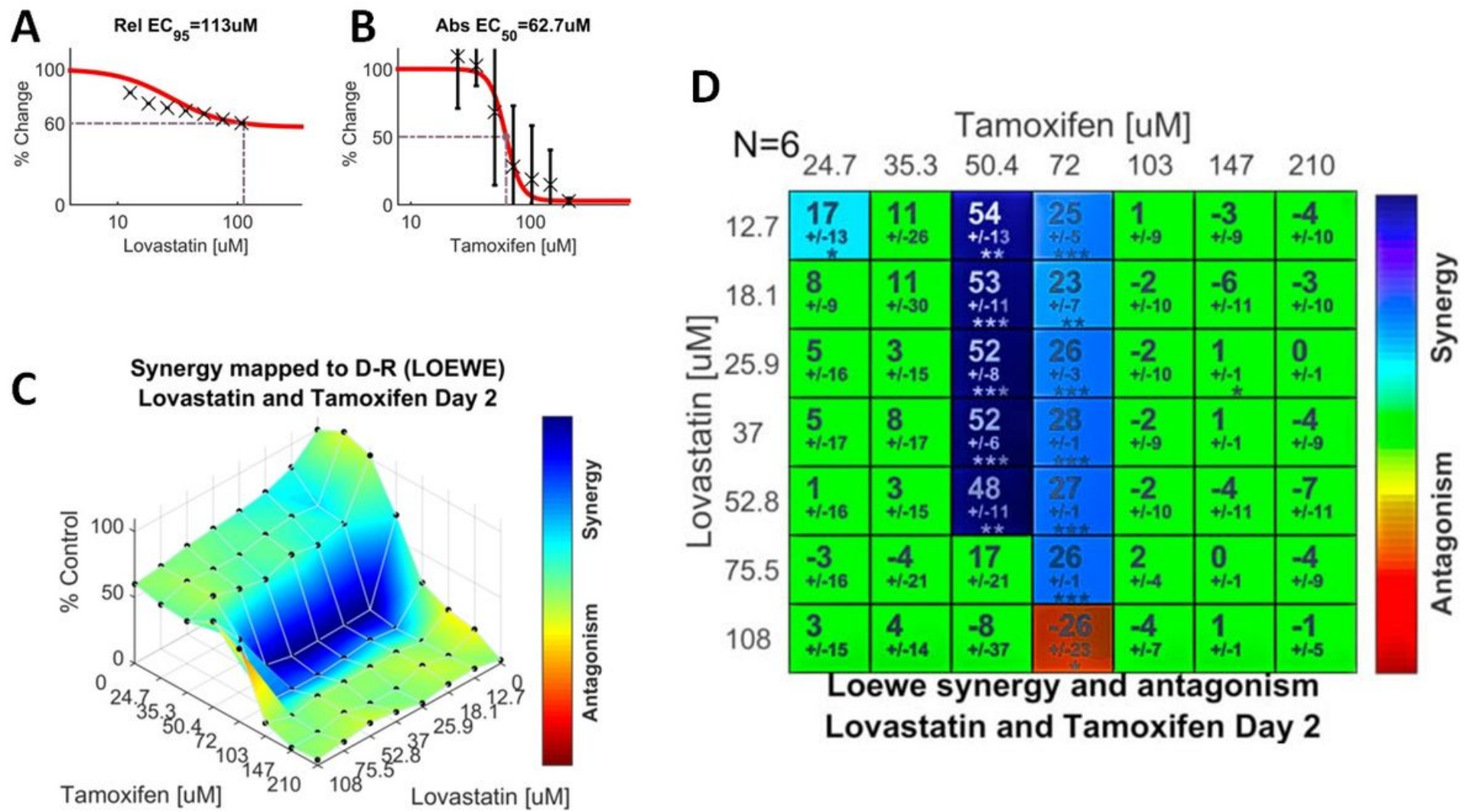

\section{Figure 2}

Strong synergism between tamoxifen and lovastatin; results of the dual-drug assay of tamoxifen plus lovastatin on Day 2. The data from six biologic replicates are included in the calculations. A. Single agent dose-response data and fitted curve for lovastatin, calculated from the normalized cell density data recorded from Column 9 of the 96 -well plate (see Figure 1). In the case of lovastatin, data from 49 experiments are aggregated to generate the curve (see Methods). B. Single agent dose-response data and fitted curve for tamoxifen ( $N=6$ ), generated from normalized Column 10 data (Figure 1). The Loewe model generates the reference concentration space from the two single agent dose-response curves. C. Shown here is the experimental two-drug combination dose-response surface, expressed as a percentage of the control value, calculated from the cells-only data and normalized to a value of 1 (see Methods). The plot thus portrays the efficacy of each of the 49 dual-drug combinations. Overlain on the dose-response surface are the Loewe synergy levels depicted in (D), colored without regard to statistical significance, according to the Synergism/Antagonism scale at the right of the figure. D. Shown in matrix format are synergy levels calculated according to the Loewe additivity model from the dual-drug experimental dose-response in comparison to the reference dose-response surfaces. The larger numeral in each box is the synergy score; negative values indicate antagonism. Boxes are coloured green if the synergy score is not significant. The boxes coloured according to the Synergism/Antagonism scale indicate results that are statistically significant by the one-sample t-test. The degrees of significance are as follows: * $p<5 \times 10-2 ;{ }^{* \star} p<10-3$, $\star \star \star p<10-4$. The number of biologic replicates $(\mathrm{N})$ is indicated at the top left of the matrix. 

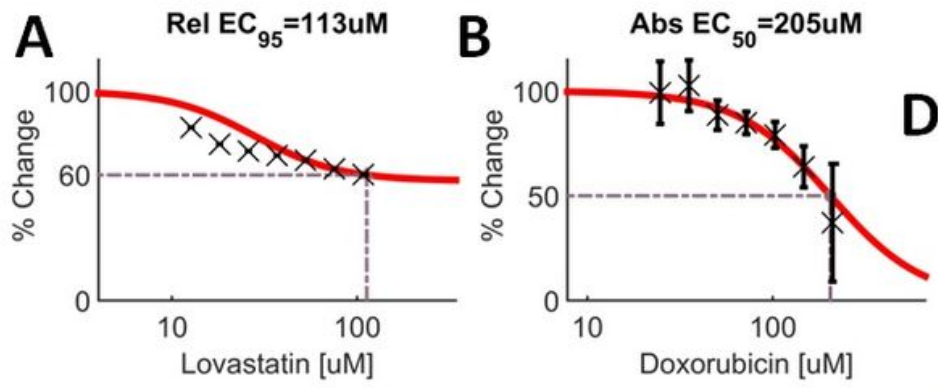

C

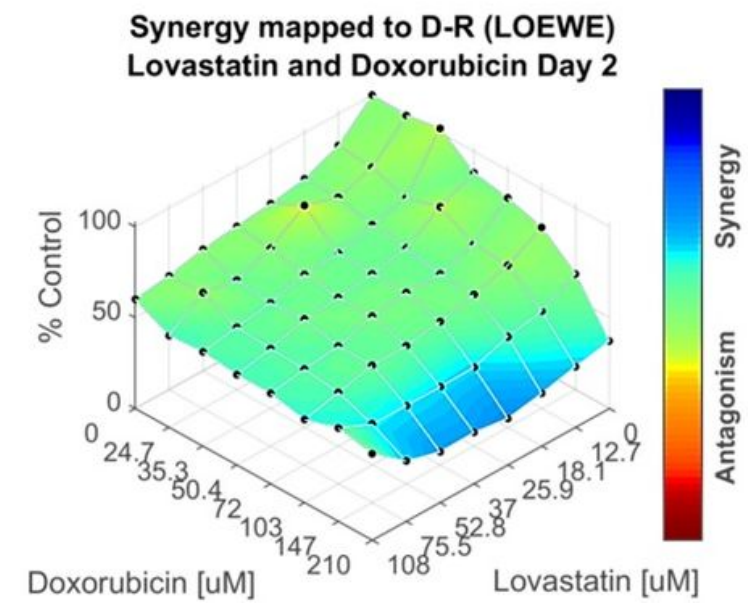

D

\begin{tabular}{|c|c|c|c|c|c|c|c|}
\hline \multirow{2}{*}{$N=$} & \multicolumn{6}{|c|}{ Doxorubicin [uM] } & \multirow[b]{2}{*}{210} \\
\hline & 24.7 & 35.3 & 50.4 & 72 & 103 & 147 & \\
\hline 12.7 & $\begin{array}{l}-3 \\
+/-16\end{array}$ & $\underset{+1-9}{2}$ & $\begin{array}{l}-6 \\
+/-10\end{array}$ & $\begin{array}{c}1 \\
+1-9\end{array}$ & $\begin{array}{l}-2 \\
+1-4\end{array}$ & $\underset{+l-16}{6}$ & $\begin{array}{c}15 \\
+\mid-21\end{array}$ \\
\hline 18.1 & $\begin{array}{l}-1 \\
+1.7\end{array}$ & $\begin{array}{l}3 \\
+1-9\end{array}$ & $\begin{array}{l}3 \\
+l-10\end{array}$ & $\begin{array}{l}4 \\
+1-9\end{array}$ & 2 & $\begin{array}{l}9 \\
+1-10\end{array}$ & 20 \\
\hline 5.9 & $\begin{array}{l}-10 \\
+/-21\end{array}$ & $\underbrace{}_{+1-10}$ & 4 & 2 & $\begin{array}{l}4 \\
+l-13\end{array}$ & $\begin{array}{c}15 \\
+/-13 \\
-\end{array}$ & 23 \\
\hline 37 & $\begin{array}{l}-1 \\
+/-16\end{array}$ & $\begin{array}{l}3 \\
+l-11\end{array}$ & $\begin{array}{l}4 \\
+i-12\end{array}$ & ${ }_{+/-12}^{2}$ & $\begin{array}{l}6 \\
+/-12 \\
\end{array}$ & $\begin{array}{l}15 \\
+/-13 \\
*\end{array}$ & 21 \\
\hline 52.8 & $\begin{array}{l}4 \\
+/-16\end{array}$ & $\underbrace{}_{+/-13}$ & $\begin{array}{l}3 \\
+1-14\end{array}$ & $\underbrace{}_{+1-16}$ & $\begin{array}{l}5 \\
+/-15\end{array}$ & $\begin{array}{c}14 \\
+l-12 \\
*\end{array}$ & 21 \\
\hline 75.5 & $\begin{array}{l}-2 \\
+/-15\end{array}$ & $\begin{array}{l}6 \\
+l-16\end{array}$ & $\begin{array}{l}7 \\
+l-17\end{array}$ & $\begin{array}{l}7 \\
+1-16\end{array}$ & $\begin{array}{l}8 \\
+l-17\end{array}$ & ${ }_{+l-18}^{13}$ & $\begin{array}{c}16 \\
+l-14\end{array}$ \\
\hline 108 & $\begin{array}{l}9 \\
+/-16\end{array}$ & $\begin{array}{l}8 \\
+/-13\end{array}$ & $\begin{array}{l}9 \\
+\mid-17\end{array}$ & $\begin{array}{l}9 \\
+\mid-14\end{array}$ & $\begin{array}{c}12 \\
+/-15\end{array}$ & $\begin{array}{l}5 \\
+/-16\end{array}$ & $\begin{array}{l}1 \\
+/-15\end{array}$ \\
\hline
\end{tabular}

Loewe synergy and antagonism Lovastatin and Doxorubicin Day 2

\section{Figure 3}

Dual drug assays of doxorubicin and lovastatin $(\mathrm{N}=7)$ analyzed by the Loewe additivity model. A. and $B$. Single agent dose-response data and fitted curves compiled for lovastatin and doxorubicin, respectively. C. Efficacy of the dual-drug combinations overlain with synergy scores. D. Synergy levels calculated by the Loewe model and colored when significant. Only the highest two concentrations of doxorubicin exhibited statistically significant interactions with lovastatin. See the legend of Figure 3 for details of the plots shown. 

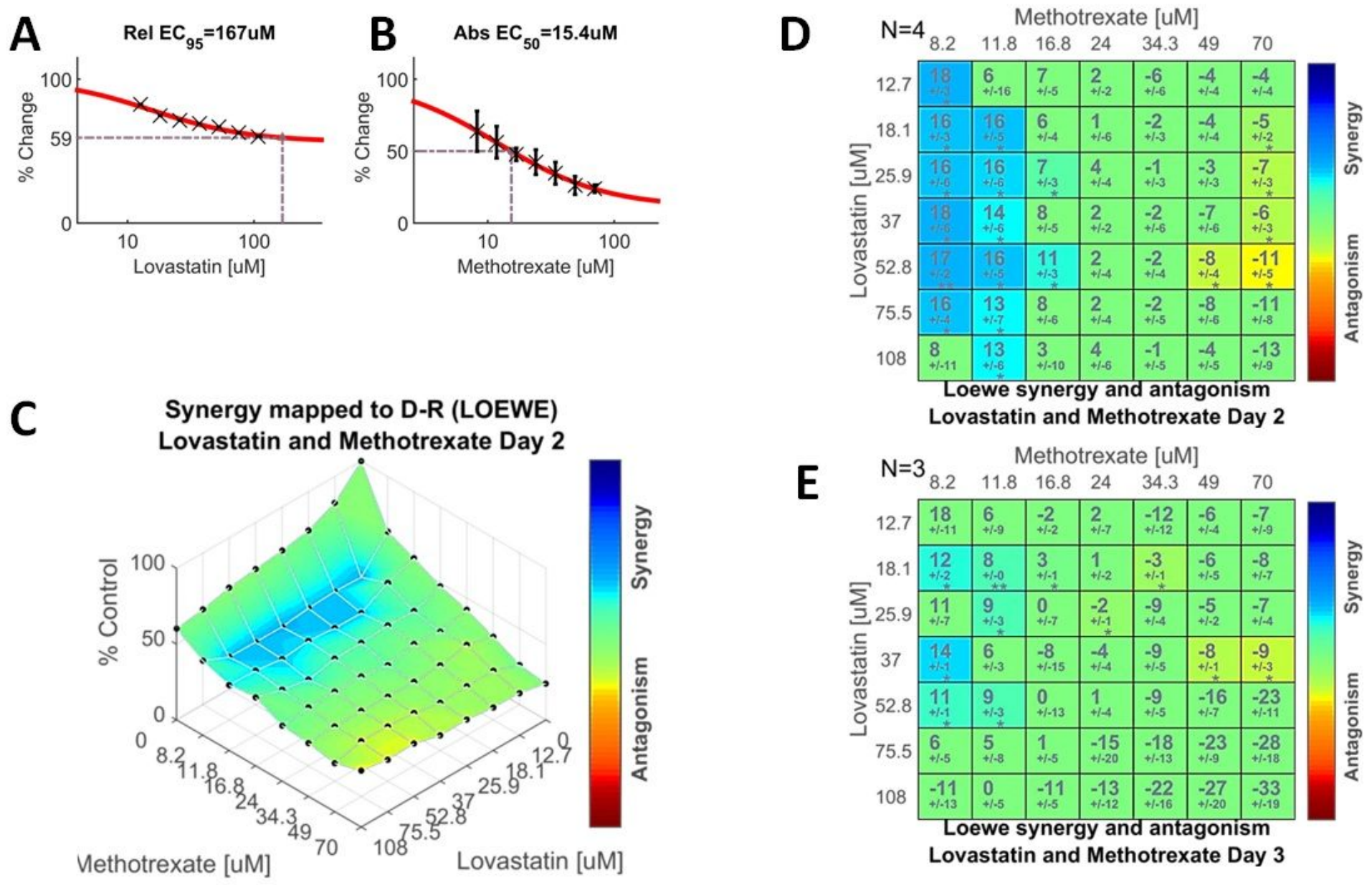

Figure 4

Lower concentrations of methotrexate synergize with the entire range of lovastatin concentrations tested.

Shown are the single agent dose-response curves for lovastatin (A) and methotrexate (B). The experimental dose-response surface, $C$, illustrates the efficacy of the dual-drug combinations and is overlain with synergy and antagonism according to the Loewe model. D. The Loewe synergy and antagonism matrix on day two $(\mathrm{N}=4)$ indicates the statistically significant synergy populating the concentration space framed by the lower concentrations of methotrexate and nearly the entire concentration range of lovastatin. Significant antagonism is detected only with the highest two concentrations of methotrexate. E. On day three $(\mathrm{N}=3)$, significant synergism and antagonism is less evident although the overall pattern of interactions is similar to that on day two. A detailed description of the various plots is given in Figure 3 . 
A
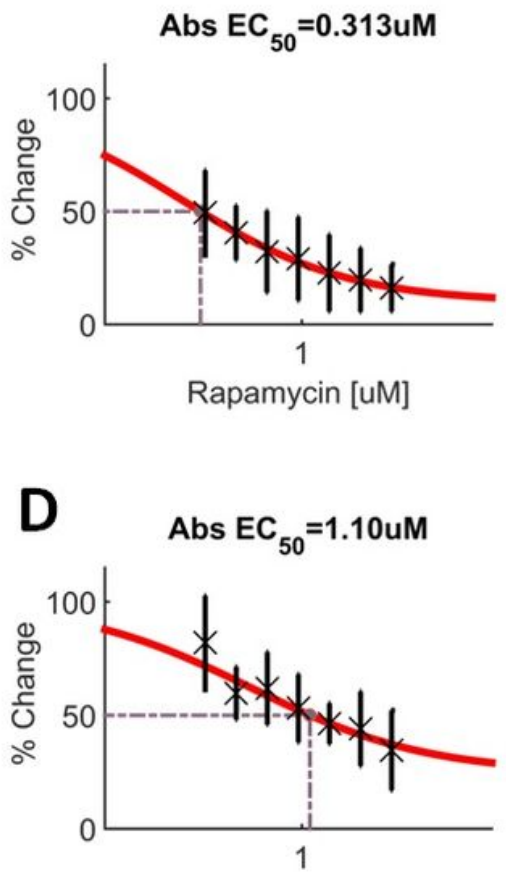

Rapamycin [uM]
B

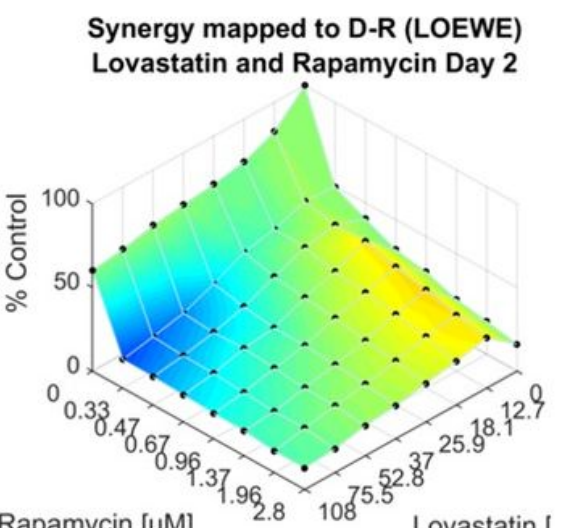

Synergy mapped to D-R (LOEWE)

$\mathbf{E}$

흘

Lovastatin Versus Rapamycin Day 3



Rapamycin [uM]

C
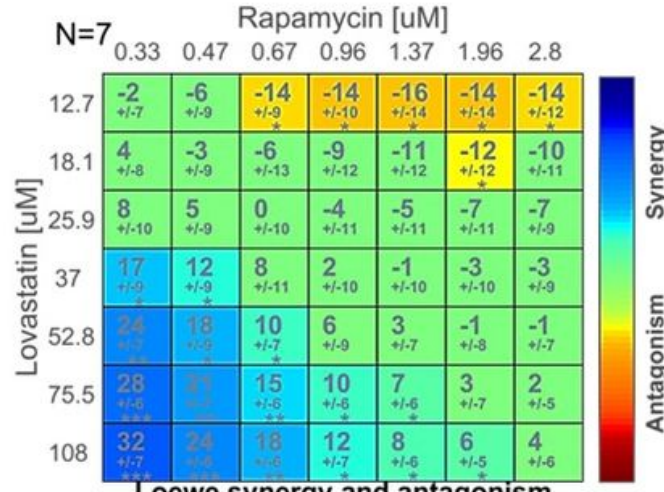

Loewe synergy and antagonism Lovastatin and Rapamycin Day 2

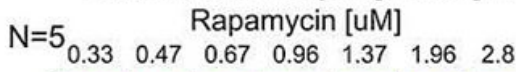

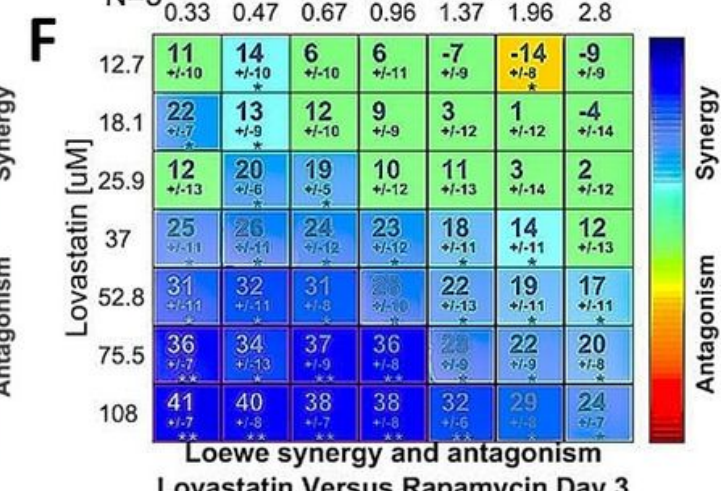

Lovastatin Versus Rapamycin Day 3

\section{Figure 5}

Drug interactions of rapamycin and lovastatin on days two $(N=7)$ and three $(N=5)$, indicating profound synergy between the two agents. The single agent dose-response curve for rapamycin is shown for day two (A) and day three (D). Note that the EC50 triples on day three. B. Rapamycin combined with lovastatin has substantial efficacy over much of the dose-response concentration space on day two. E. The doseresponse space is similar on day three, despite the shift in the single agent dose-response curve for rapamycin (see D). The Loewe synergy matrices on day two (C) and day three (F) are similar but with an even greater extent of statistically significant synergistic interactions at the later time point. The presence of strong antagonism, antipodal to the synergistic interactions, resulted in a modest global SUM_SYN_ANT score (Figure 2A) but the global metric reverses on day 3 (Figure 2B) because of the expanding synergism and weakening antagonism $(\mathrm{F})$. 
A

$\mathrm{N}=7$ 5-FU [ug/ul]

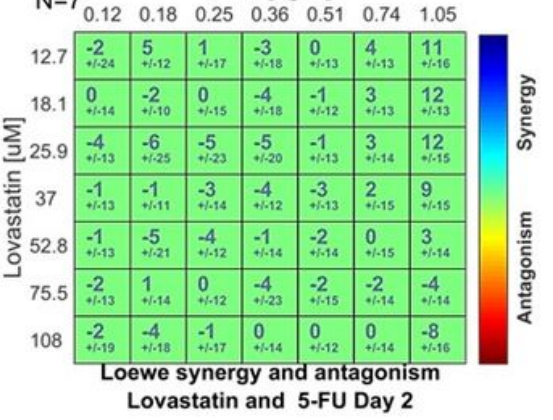

D



B

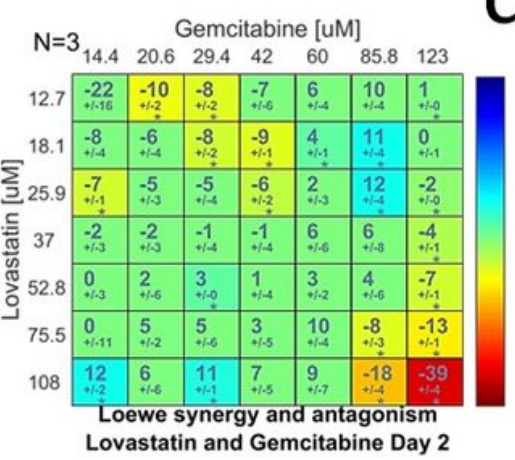

E

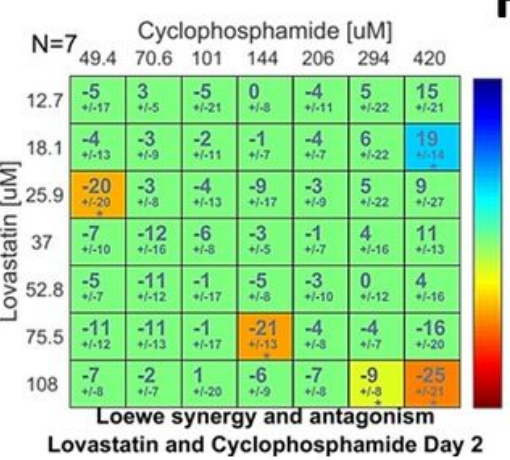

C



$\mathbf{F}$



Figure 6

Loewe additivity matrices on day two of 5-fluorouracil (A), gemcitabine (B), epothilone (C), cisplatin (D), cyclophosphamide $(E)$, and etoposide $(F)$. The global metrics were neutral or antagonistic for all six drugs in combination with lovastatin. The number of biologic replicates is specified in each panel.
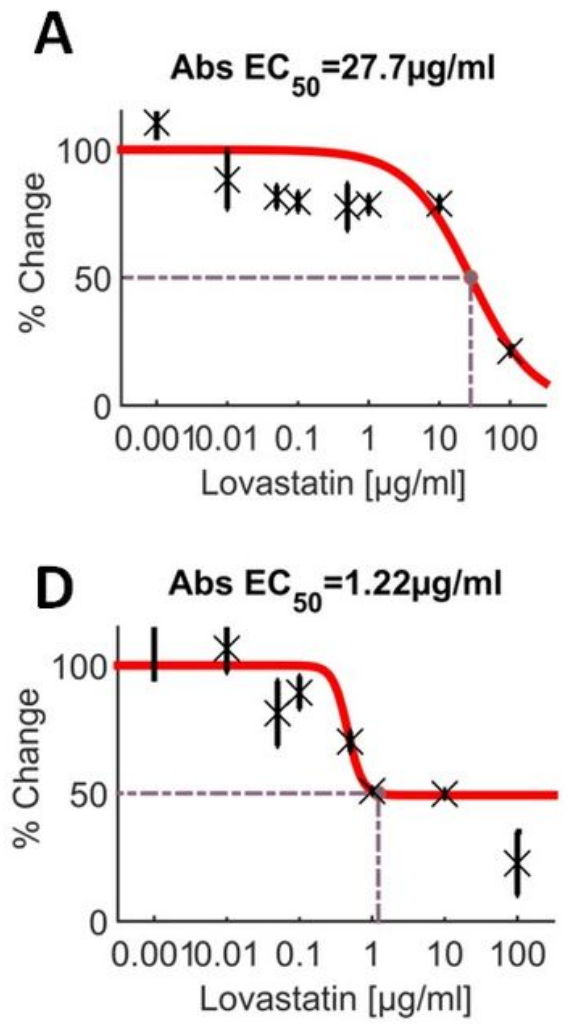
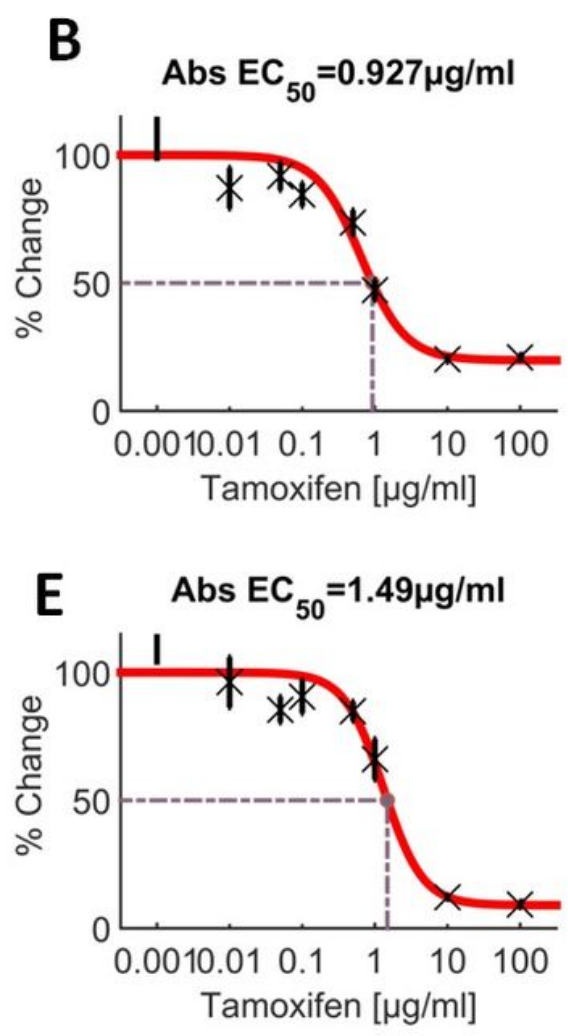
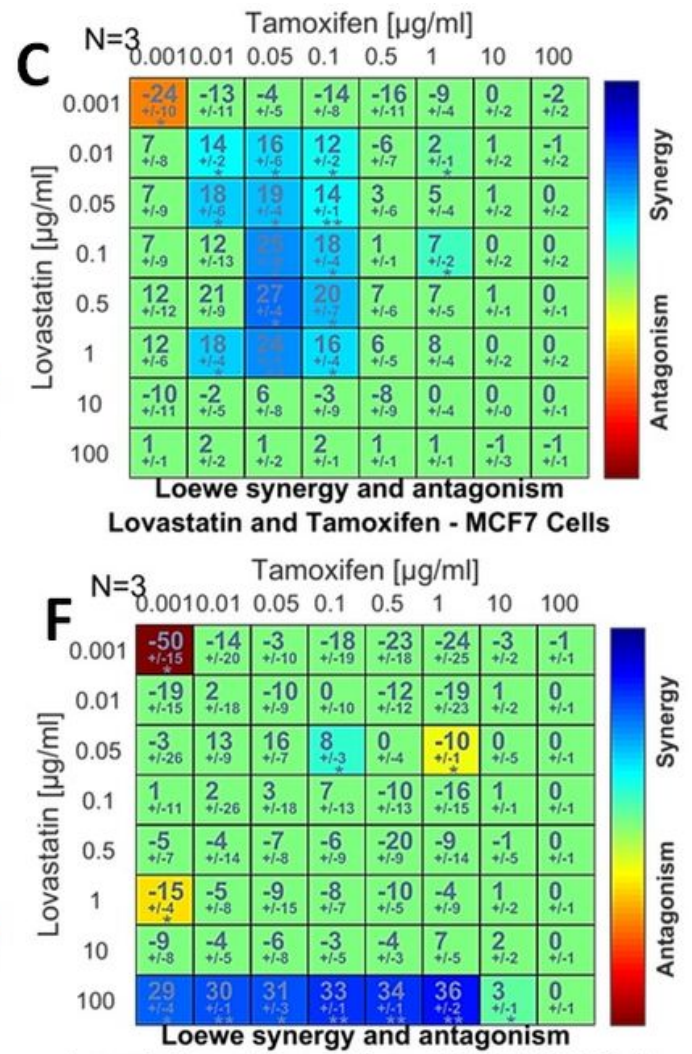

Lovastatin and Tamoxifen - MDA-MB-231 Cells 


\section{Figure 7}

Tamoxifen and lovastatin dual-drug assays in human breast cancer cell lines, MCF7 (A - C), MDA-MB-231 $(D-F)$, and SKBR3 $(G-I)$, and lung adenocarcinoma $(J-L)$. Shown are the single agent dose response data and the Loewe additivity model matrices. Note the variability of the lovastatin dose responses between cell lines $(A, D, G, J)$. The tamoxifen single agent dose responses vary to a lesser degree $(B, E, H, K)$; the EC50 values are clustered about the $1 \mu \mathrm{g} / \mathrm{ml}$ concentration. The Loewe synergy matrices $(C, F, I, L)$, all of which include significant synergism and antagonism, are quite dissimilar. The concentration space of the estrogen receptor-positive MCF7 cell line (C) resembles that obtained with S. cerevisiae (see Figure 3D). $\mathrm{N}=3$ for each cell line.

\section{Supplementary Files}

This is a list of supplementary files associated with this preprint. Click to download.

- SupplementalTABLE1 revisionmay.xlsx

- AdditionalFile2.pdf

- AdditionalFile1.pdf

- FigureS1.pdf

- FigureS5.pdf

- FigureS3.pdf

- Figures2.pdf

- FigureS4.pdf

- FigureS7.pdf

- FigureS6.pdf 